\title{
Prof. Dr. habil. Ernst Ritter
}

75 Jahre

Am 14. April 2004 vollendet der Chefredakteur unserer Zeitschrift Prof. Dr. habil. ERNST RITTER sein 75. Lebensjahr. Dazu gratulieren ihm seine Freunde und Weggefährten, viele Kollegen aus der Wissenschaft und Praxis, seine Schüler und ehemaligen Mitarbeiter.

Im schlesischen Schweidnitz am 14. 4. 1929 geboren, musste er die Oberrealschule im Alter von 16 Jahren für den Kriegsdienst verlassen. Nach Kriegsende absolvierte er in Tierzuchtbetrieben Sachsens und Thüringens eine landwirtschaftliche Berufsausbildung, die 1949 mit dem „Staatlich geprüften Landwirt“ in Eisenach abgeschlossen wurde. Es folgte 1952 das Landwirtschaftsstudium an der Friedrich-Schiller-Universität Jena. Danach arbeitete er acht Jahre als Assistent und Oberassistent bei seinem von ihm hochverehrten Lehrer FRITZ HOFMANN am Institut für Tierzucht und Milchwirtschaft in Jena, bei dem er auch 1956 promovierte. Von 1960 bis 1964 war er Leiter des landwirtschaftlichen Beratungsinstitutes in Tautenhain/Thüringen. In dieser Zeit leitete er auch im Rahmen eines Forschungsauftrages am Jenaer Tierzuchtinstitut die Umzüchtungsarbeiten des Deutschen veredelten Landschweines zum Fleischschwein und habilitierte sich 1966 mit einer Arbeit zu dieser Thematik an der Landwirtschaftlichen Fakultät der Friedrich-Schiller-Universität Jena. Im Jahr 1964 wurde er zum Dozenten für Tierzucht an die Hochschule für Landwirtschaft Bernburg berufen. Im Jahr 1967 legte er die staatliche Tierzuchtleiterprüfung ab. In dieser Zeit hat ERNST RITTER nachhaltig an der Entwicklung der nationalen und internationalen Schweinezucht mitgewirkt, so z. B. bei der Fleischschweinzüchtung, der Umrüstung von Mastanstalten zu Gruppenprüfanstalten oder der Entwicklung des Stichprobentestes. Im Jahre 1970 übernahm er die Leitung der Abteilung Schweinezucht des Forschungszentrums für Tierproduktion DummerstorfRostock. Will man das wissenschaftliche Schaffen des Jubilars in dieser Zeit in einem für eine Laudatio darstellbaren Umfang würdigen, sind folgende Schwerpunkte, an denen er mit seinen Mitarbeitern gearbeitet hat, besonders hervorzuheben: Mitgestaltung des Hybridschweinezuchtprogramms in der DDR, zuchtmethodische Arbeiten, darunter langjährige Selektionsexperimente in großen Zuchtbetrieben zu RS und RRS beim Schwein, Entwicklung komplexer Selektionsindizes unter Einbeziehung der Informationen aus allen Leistungsprüfungen und deren Umsetzung in der Schweinezüchtung, Antagonismen zwischen speziellen Leistungen und Fitness sowie züchterische Möglichkeiten zu ihrer Vermeidung, züchterische Verbesserung der reproduktiven Fitness, insbesondere der Wurfleistung, Analyse von Schweinezuchtpopulationen hinsichtlich genetischer und phänotypischer Parameter sowie die genetische Relevanz der Fruchtbarkeit von Besamungsebern. Dieses umfassende Werk 
des Jubilars ist in mehr als 200 wissenschaftlichen Veröffentlichungen, Büchern und Buchbeiträgen dokumentiert. Das in seiner Vita auszumachende Gespür für den Praxisbezug, findet sich in seinen Forschungsarbeiten, Publikationen oder Vorträgen und dürfte wohl mit den praktischen Arbeiten in der Phase der Berufsfindung, seiner Erfahrung in der Tierzuchtberatung und seinem Lehrer FRITZ HOFMANN begründet sein.

Im Jahre 1976 wurde ERNST RITTER durch die Akademie der Landwirtschaftswissenschaften zu Berlin zum Professor berufen. Nach der Wiedervereinigung Deutschlands war er bis zu seiner Emeritierung 1994 im Forschungsbereich „Populationsbiologie und Züchtungsforschung“ des neugegründeten Forschungsinstitutes für die Biologie landwirtschaftlicher Nutztiere Dummerstorf tätig.

Während seiner Vorlesungstätigkeit in Jena, Bernburg und Rostock begeisterte er viele seiner Studenten für sein Fach, war seinen Schülern stets ein anspruchsvoll kritischer, aber hilfsbereiter und fördernder Mentor. Viele seiner 19 Doktoranden und 4 Habilitanden zählen heute zu seinen Gratulanten. Eine unverkennbare Bedeutung hat die Vorbildwirkung von ERNST RITTER, sie wurde fast zum Anspruch, wenn auch nie direkt. Das galt sowohl für seine Lehrals auch Leitungstätigkeit, wo er es verstanden hat, trotz aller Disziplin, die freie Entfaltung und die kreativen Bestrebungen der Mitarbeiter voll zu unterstützen. Kennzeichnend für ihn ist auch sein den Mitarbeitern stets angemahntes Motto, dass Wissenschaftler die Pflicht zur Veröffentlichung ihrer Ergebnisse haben und diese nur dann erfolgreich angewendet werden können, wenn sie verständlich mitgeteilt werden. Mit dieser Haltung konnte es nicht ausbleiben, dass er 1979 die Schriftleitung des „Archiv für Tierzucht“ übernahm und diese noch heute mit großem Engagement und Begeisterung ausübt. Mit gewohnter Energie, Umsicht und Hingabe prägt er seit nunmehr 25 Jahren als Schriftleiter gemeinsam mit dem Redaktionskollegium den Inhalt dieser Zeitschrift und hat maßgeblichen Anteil an deren stetig steigender nationaler und internationaler Akzeptanz. Den damit verbundenen Herausforderungen wie Management, Kontaktaufbau und -pflege weit über die Landesgrenzen hinaus, hat sich ERNST RITTER mit außergewöhnlichem Einsatz bis in die Gegenwart gestellt, wofür ihm herzlich gedankt sei. Damit hat er sich tatkräftig, entscheidend und erfolgreich um die institutionelle Konsolidierung und die wissenschaftlich-inhaltliche Weiterentwicklung der Zeitschrift verdient gemacht. Seine Bestrebungen um die Aufnahme englischsprachiger Beiträge in diese Zeitschrift ermöglichten den Zugang zu neuen Leserkreisen. Er öffnete das „Archiv für Tierzucht“ für Autoren aus ganz Europa und Übersee um internationale wissenschaftliche Kommunikation und den Austausch von Forschungsergebnissen zu fördern. Sein Sachverstand, seine Zielstrebigkeit und Ausdauer, seine liebenswürdige, verbindliche Art, verbunden mit Redlichkeit, kamen ihm dabei zugute. Sie machen ihn zu einem anerkannten Gesprächspartner für die Autoren und für seine Fachkolleginnen und -kollegen im Redaktionskollegium. Die Verdienste, die sich der Jubilar um diese Zeitschrift erworben hat, können schon heute als bedeutend für die Tierzuchtwissenschaft charakterisiert werden. Neben den Tagesaufgaben, widmet er sich, als einer der Gründungsväter, mit Engagement dem Aufbau und der Weiterentwicklung des „Deutschen Schweinemuseums“ in Ruhlsdorf, welches inzwischen ein historischer Anziehungspunkt für viele Schweinezüchter und Gäste wurde.

Viele, die den Weg von ERNST RITTER kreuzten, werden gern an diese Begegnungen zurückdenken. Mit Eigenschaften wie fachliche Kompetenz, Kooperationsbereitschaft, seinem guten Rat, seiner freundlichen Offenheit, seiner Hilfsbereitschaft und dem Sinn für Geselligkeit wird ein großer Freundeskreis fast unvermeidlich. Möge sich der Jubilar noch lange Zeit dieser Wertschätzung und Freundschaft erfreuen. Wir, Kollegen und Tierzüchter, haben allen Anlass uns bei dem Jubilar für sein mehr als 50jähriges Wirken im Dienst der Tierzucht zu bedanken. Für die kommenden Jahre wünschen die ihm verbundenen Freunde, Kollegen, Züchter, Schüler und der Laudator alles Gute, vor allem Gesundheit und noch viele schöne Jahre im Kreise seiner Familie. 